\title{
Modulation of the Singlet-Singlet Through Space Energy Transfer Rates in Cofacial Bisporphyrin and Porphyrin-Corrole Dyads
}

Claude P. Gros, Frédéric Brisach, Anastasia Meristoudi, Enrique Espinosa Roger Guilard and Pierre D. Harvey

\section{SUPPORTING MATERIAL}

\section{Table of contents}

\begin{tabular}{|c|c|}
\hline Experimental & $\begin{array}{l}\text { Instrumentation, synthetic procedures and analytical data of } \\
\left(\mathrm{Mes}_{2} \mathrm{Ph}_{1} \mathrm{Cor}\right) \mathrm{H}_{3}(\mathbf{6}) .\left(\mathrm{PMes}_{2} \mathrm{CO}\right) \mathrm{ZnH}_{3}(\mathbf{1 4}),\left(\mathrm{PMes}_{2} \mathrm{COx}\right) \mathrm{ZnH}_{3} \quad(\mathbf{1 3}) \text {, } \\
(\mathrm{DPOx}) \mathrm{ZnH}_{2}(\mathbf{1 7}),(\mathrm{DPOx}) \mathrm{Zn}_{2}(\mathbf{1 8}) .\end{array}$ \\
\hline Figure S1 & $\begin{array}{l}\text { Top and side views of the optimized geometry for }\left(\mathrm{PMes}_{2} \mathrm{CX}\right) \mathrm{ZnH}_{3}(\mathbf{1 5}) \text {. } \\
\text { (top) and for }\left(\mathrm{PMes}_{2} \mathrm{CO}\right) \mathrm{ZnH}_{3}(\mathbf{1 4}) \text { (bottom). }\end{array}$ \\
\hline Figure S2 & $\begin{array}{l}\text { UV-vis spectra of }\left(\mathrm{Et}_{4} \mathrm{Me}_{4} \mathrm{Ph}_{1} \mathrm{P}\right) \mathrm{H}_{2}(\mathbf{7}) \text { as compared to the free base } \\
\text { bisporphyrin (DPOx) } \mathrm{H}_{4}(\mathbf{1 6}) \text {. }\end{array}$ \\
\hline Figure S3 & $\begin{array}{l}\text { UV-vis spectra of }\left(\mathrm{Et}_{4} \mathrm{Me}_{4} \mathrm{Ph}_{1} \mathrm{P}\right) \mathrm{Zn}(\mathbf{8}) \text { as compared to the dizinc } \\
\text { bisporphyrin }(\mathrm{DPOx}) \mathrm{Zn}_{2}(\mathbf{1 8}) \text {. }\end{array}$ \\
\hline Figure S4 & $\begin{array}{l}\mathrm{UV} \text {-vis spectra of }\left(\mathrm{Mes}_{2} \mathrm{Ph}_{1} \mathrm{Cor}\right) \mathrm{H}_{3}(\mathbf{6})+\left(\mathrm{Et}_{4} \mathrm{Me}_{4} \mathrm{Ph}_{1} \mathrm{P}\right) \mathrm{Zn}(\mathbf{8}) \text { as } \\
\text { compared to the monozinc derivative porphyrin-corroles } \\
\left(\mathrm{PMes}_{2} \mathrm{COx}\right) \mathrm{ZnH}_{3}(\mathbf{1 3}),\left(\mathrm{PMes}_{2} \mathrm{CO}\right) \mathrm{ZnH}_{3}(\mathbf{1 4}) \text { and }\left(\mathrm{PMes}_{2} \mathrm{CX}\right) \mathrm{ZnH}_{3}(\mathbf{1 5}) \text {. }\end{array}$ \\
\hline Figure S5 & $\begin{array}{l}\text { Fluorescence spectra of }\left(\mathrm{PMes}_{2} \mathrm{CO}\right) \mathrm{ZnH}_{3}(\mathbf{1 4}) \text { (blue) }\left(\mathrm{Mes}_{2} \mathrm{Ph}_{1} \mathrm{Cor}\right) \mathrm{H}_{3} \\
\text { (6) (black) and (OEP)Zn (red) in 2-MeTHF at } 77 \mathrm{~K} \text {. }\end{array}$ \\
\hline Ref. 29 & Complete Ref. 29 \\
\hline
\end{tabular}




\section{Experimental Section SI}

Instrumentation. ${ }^{1} \mathrm{H}$ NMR spectra were recorded on a Bruker DRX-500 AVANCE transform spectrometer at the "Centre de Spectrométrie Moléculaire de l'Université de Bourgogne"; chemical shifts are expressed in ppm relative to chloroform (7.26 ppm) or benzene $(7.15 \mathrm{ppm})$. Microanalyses were performed at the Université de Bourgogne on a Fisons EA 1108 CHNS instrument. UV-visible spectra were recorded on a Varian Cary 1 spectrophotometer. Mass spectra were obtained on a Bruker ProFLEX III spectrometer in the MALDI / TOF mode using dithranol as a matrix. Emission and excitation spectra were obtained using a double monochromator Fluorolog 2 instrument from Spex. Fluorescence lifetimes were measured on a Timemaster Model TM-3/2003 apparatus from PTI. The source was nitrogen laser with high-resolution dye laser (FWHM 1500 ps) and the fluorescence lifetimes were obtained from deconvolution or distribution lifetimes analysis.

10-Phenyl-5,15-dimesitylcorrole $\left(\mathbf{M e s}_{2} \mathbf{P h}_{1} \mathbf{C o r}\right) \mathbf{H}_{3}$ (6). Benzaldehyde (0.320 g, 3.0 mmol) and 5-mesityldipyrromethane ( $1.58 \mathrm{~g}, 6.0 \mathrm{mmol}, 2$ equi.) were dissolved under argon in dichloromethane $(170 \mathrm{~mL})$. The mixture was stirred at room temperature for $5 \mathrm{~min}$, and TFA $(17 \mu \mathrm{L})$ was added. After stirring at room temperature for $5 \mathrm{~h}$, the mixture was diluted with dichloromethane (3.0 L) and a solution of DDQ (2,3-dichloro-5,6-dicyano-1,4benzoquinone) (1.30 g dissolved in $20 \mathrm{~mL}$ of THF) was added. The mixture was stirred at room temperature for $30 \mathrm{~min}$, concentrated and filtered through a silica pad using $\mathrm{CH}_{2} \mathrm{Cl}_{2} /$ heptane (70/30) as eluent. After evaporation, the resulting solid was chromatographed twice on silica gel using $\mathrm{CH}_{2} \mathrm{Cl}_{2}$ /heptane (1/1) as eluent for the first chromatography and $\mathrm{CH}_{2} \mathrm{Cl}_{2}$ /heptane (1/6) as eluent for the second chromatography. After recrystallization in a $\mathrm{CH}_{2} \mathrm{Cl}_{2}$ /heptane mixture, the title compound (6) was obtained in $5 \%$ yield $(91.5 \mathrm{mg})$ as a dark violet solid. ${ }^{1} \mathrm{H}$ NMR $\left(\mathrm{CDCl}_{3}\right) \delta=-1.92$ (br. s, 3H, NH), 1.90 (s, 12H), $2.57(\mathrm{~s}, 6 \mathrm{H}), 7.14-7.31(\mathrm{~m}, 5 \mathrm{H}), 7.23(\mathrm{~s}, 4 \mathrm{H}) ; 8.30(\mathrm{~d}, J=4,04 \mathrm{~Hz}, 2 \mathrm{H}), 8.44-8.49(\mathrm{~m}$, 
4H), $8.86(\mathrm{~d}, J=4,04 \mathrm{~Hz}, 2 \mathrm{H}) . \mathrm{SM}(\mathrm{MALDI} / \mathrm{TOF}): \mathrm{m} / \mathrm{z}=610.10[\mathrm{M}]^{+\bullet} ; 610.79$ calcd. for $\mathrm{C}_{43} \mathrm{H}_{38} \mathrm{~N}_{4}$. UV/Vis $\left(\mathrm{CH}_{2} \mathrm{Cl}_{2}\right): \lambda_{\max }\left(\varepsilon \times 10^{-3} \mathrm{Lmol}^{-1} \mathrm{~cm}^{-1}\right)=407$ (117.0), 425 (89.9), 567 (16.8), 604 (11.8), 637 (6.3). Anal. Calcd for $\mathrm{C}_{43} \mathrm{H}_{38} \mathrm{~N}_{4}$ : C, 84.56; H, 6.27; N, 9.17. Found: C, 84.18; H, 5.91; N, 8.86.

\section{[13,17-Diethyl-5-(4-formyl-diphenylether)-2,3,7,8,12,18-hexamethylporphyrin]}

(9).

Under nitrogen and with the exclusion of light, a mixture of 5,4-diformyl-diphenylether (2.25 g, 9.96 mmol, 1 equiv.) and 1,19-dideoxy-8,12-diethyl-2,3,7,13,17,18-hexamethylbiladien-a,c (6.00 g, $9.96 \mathrm{mmol}, 1$ equiv.) was dissolved in ethanol $(1000 \mathrm{~mL})$. A solution of paratoluenesulfonic acid monohydrate PTSA (10.00 g, $52.7 \mathrm{mmol}, 5.3$ equiv.) dissolved into absolute ethanol $(250 \mathrm{~mL})$ was added during $16 \mathrm{~h}$ using a peristaltic pump. The reaction mixture was stirred under reflux for $24 \mathrm{~h}$. The mixture was cooled to room temperature and the solvent was evaporated. The residue so obtained was dissolved in dichloromethane (500 mL) neutralized with a saturated solution of $\mathrm{NaHCO}_{3}$, and then washed thoroughly with water. The organic phase was dried over $\mathrm{MgSO}_{4}$ and filtered, and the solvent was removed under vacuum. The residue obtained was chromatographed on a silica column $\left(\mathrm{CH}_{2} \mathrm{Cl}_{2}\right.$ as eluent) to yield (9) as a purple solid (1.540 g, $2.38 \mathrm{mmol}, 24 \%$ yield). ${ }^{1} \mathrm{H}$ NMR $\left(\mathrm{CDCl}_{3}\right) \delta=-$ $3.30\left(\mathrm{~s}, 1 \mathrm{H}, \mathrm{NH}\right.$-porphyrin), $-3.14\left(\mathrm{~s}, 1 \mathrm{H}, \mathrm{NH}\right.$-porphyrin), $1.87\left(\mathrm{t}, 6 \mathrm{H},{ }^{3} \mathrm{~J}_{\mathrm{H}-\mathrm{H}}=7.67 \mathrm{~Hz}\right.$, $\mathrm{CH}_{2} \mathrm{CH}_{3}$-porphyrin), 2.67 (s, $6 \mathrm{H}, \mathrm{CH}_{3}$-porphyrin), 3.59 (s, 6H, $\mathrm{CH}_{3}$-porphyrin), 3.63 (s, $6 \mathrm{H}$, $\mathrm{CH}_{3}$-porphyrin), 4.05 (q, $4 \mathrm{H},{ }^{3} \mathrm{~J}_{\mathrm{H}-\mathrm{H}}=7.70 \mathrm{~Hz}, \mathrm{CH}_{2} \mathrm{CH}_{3}$-porphyrin), $6.88\left(\mathrm{t}, 1 \mathrm{H},{ }^{3} \mathrm{~J}_{\mathrm{H}-\mathrm{H}}=7.49\right.$ $\mathrm{Hz}, \mathrm{H}$-diphenylether), $7.20\left(\mathrm{~d}, 1 \mathrm{H},{ }^{3} \mathrm{~J}_{\mathrm{H}-\mathrm{H}}=9.0 \mathrm{~Hz}, \mathrm{H}\right.$-diphenylether $), 7.27\left(\mathrm{~d}, 1 \mathrm{H},{ }^{3} \mathrm{~J}_{\mathrm{H}-\mathrm{H}}=9.55\right.$ $\mathrm{Hz}, \mathrm{H}$-diphenylether), 7.38 (m, 2H, H-diphenylether), $7.55\left(\mathrm{td}, 1 \mathrm{H},{ }^{3} \mathrm{~J}_{\mathrm{H}-\mathrm{H}}=7.39 \mathrm{~Hz}, \mathrm{H}-\right.$ diphenylether), $7.76\left(\mathrm{td}, 1 \mathrm{H},{ }^{3} \mathrm{~J}_{\mathrm{H}-\mathrm{H}}=8.05 \mathrm{~Hz},{ }^{4} \mathrm{~J}_{\mathrm{H}-\mathrm{H}}=1.58 \mathrm{~Hz}, \mathrm{H}\right.$-diphenylether), 8.12 (dd, $1 \mathrm{H}$, ${ }^{3} \mathrm{~J}_{\mathrm{H}-\mathrm{H}}=7.33 \mathrm{~Hz},{ }^{4} \mathrm{~J}_{\mathrm{H}-\mathrm{H}}=1.56 \mathrm{~Hz}, \mathrm{H}-$ diphenylether $), 9.59(\mathrm{~s}, 1 \mathrm{H}, \mathrm{CHO}), 9.94\left(\mathrm{~s}, 1 \mathrm{H}, \mathrm{H}_{\text {meso }}{ }^{-}\right.$ porphyrin), 10.16 (s, 2H, $\mathrm{H}_{\text {meso }}$-porphyrin). SM (MALDI/TOF) : m/z $=646.90[\mathrm{M}]^{+\bullet} ; 646.82$ calcd. for $\mathrm{C}_{43} \mathrm{H}_{42} \mathrm{~N}_{4} \mathrm{O}_{2}$. UV/Vis $\left(\mathrm{CH}_{2} \mathrm{Cl}_{2}\right): \lambda_{\max }\left(\varepsilon \times 10^{-3} \mathrm{Lmol}^{-1} \mathrm{~cm}^{-1}\right)=403$ (149.6), 501 
(12.1), 535 (5.10), 572 (4.51), 625 (1.35). Anal. Calcd for $\mathrm{C}_{43} \mathrm{H}_{42} \mathrm{~N}_{4} \mathrm{O}_{2}$ : C, 79.85; H, 6.54; N, 8.66. Found: C, 80.12; H, 6.88; N, 8.32.

\section{[13,17-Diethyl-5-(4-formyl-dibenzofuran)-2,3,7,8,12,18-hexamethylporphyrin]zinc}

(12). A solution of 13,17-diethyl-5-(4-formyl-dibenzofuran)-2,3,7,8,12,18hexamethylporphyrin (10) (1.00 g, 1.55 mmol, 1 equiv.), zinc acetate dihydrate $(0.680 \mathrm{~g}, 3.10$ mmol, 2 equiv.) and sodium acetate trihydrate (2.11 g, $15.50 \mathrm{mmol}, 10$ equiv.) in chloroform $(175 \mathrm{~mL}) /$ methanol $(75 \mathrm{~mL})$ mixture was heated to reflux for 30 minutes. After cooling to room temperature, dichloromethane $(300 \mathrm{~mL})$ was added. The reaction mixture was washed with water $(3 \mathrm{x} 400 \mathrm{~mL})$, dried with magnesium sulfate, filtered and the solvents were evaporated. The resulting solid was chromatographed on silica gel using first toluene as eluent and then dichloromethane to collect a pink fraction. The title compound (12) was obtained in $53 \%$ yield $(0.580 \mathrm{~g}, 0.821 \mathrm{mmol}) .{ }^{1} \mathrm{H} \mathrm{NMR}\left(\mathrm{CDCl}_{3}, 300 \mathrm{~K}\right): \delta=1.97\left(\mathrm{t}, 6 \mathrm{H}, \mathrm{CH}_{3} \mathrm{CH}_{2}-\right.$ porphyrin), 2.30 (s, 6H, $\mathrm{CH}_{3}-$ porphyrin), 3.50 (s, 6H, $\mathrm{CH}_{3}-$ porphyrin), 3.67 (s, 6H, $\mathrm{CH}_{3}-$ porphyrin), 4.00 (m, 4H, $\mathrm{CH}_{3} \mathrm{CH}_{2}$-porphyrin), 7.55 (t, 1H, H-dibenzofuran), 7.75 (t, 1H, Hdibenzofuran), 7.93 (dd, 1H, H-dibenzofuran), 8.04 (dd, 1H, H-dibenzofuran), 8.46 (dd, 1H, H-dibenzofuran), $8.50\left(\mathrm{t}, 1 \mathrm{H}, \mathrm{H}\right.$-dibenzofuran), $9.80(\mathrm{~s}, 1 \mathrm{H}, \mathrm{CHO}), 9.95\left(\mathrm{~s}, 1 \mathrm{H}, \mathrm{H}_{\text {meso }}{ }^{-}\right.$

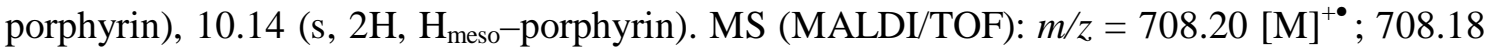
calcd. for $\mathrm{C}_{43} \mathrm{H}_{38} \mathrm{~N}_{4} \mathrm{O}_{2} \mathrm{Zn}$. UV/Vis $\left(\mathrm{CH}_{2} \mathrm{Cl}_{2}\right): \lambda_{\max }\left(\varepsilon \times 10^{-3} \mathrm{Lmol}^{-1} \mathrm{~cm}^{-1}\right)=406.0(612.8)$, 536.0 (32.1), 572.0 (29.5) nm. Anal. Calcd for $\mathrm{C}_{43} \mathrm{H}_{38} \mathrm{~N}_{4} \mathrm{O}_{2} \mathrm{Zn}$ : C, 72.93; H, 5.41; N, 7.91. Found: C, 73.28; H, 5.65; N, 7.78.

\section{[13,17-Diethyl-5-(4-formyl-diphenylether)-2,3,7,8,12,18-}

hexamethylporphyrin]zinc (11). This compound was synthesized as described above for (12), starting from 13,17-diethyl-5-(4-formyl-diphenylether)-2,3,7,8,12,18hexamethylporphyrin (9) (0.647 g, 1.036 mmol, 1 equiv.) and zinc acetate dihydrate $(0.455 \mathrm{~g}$, 2.076 mmol, 2 equiv.) in $82 \%$ yield $(0.601 \mathrm{~g}, 0.845 \mathrm{mmol}) .{ }^{1} \mathrm{H} \mathrm{NMR}\left(\mathrm{CDCl}_{3}, 300 \mathrm{~K}\right): \delta=$ 
$1.70\left(\mathrm{t}, 6 \mathrm{H}, \mathrm{CH}_{3} \mathrm{CH}_{2}\right.$-porphyrin), 2.23 (s, 6H, $\mathrm{CH}_{3}$-diphenylether), 2.80 (s, 6H, $\mathrm{CH}_{3}-$ porphyrin), 3.28 (s, $6 \mathrm{H}, \mathrm{CH}_{3}$-porphyrin), $3.36\left(\mathrm{~m}, 4 \mathrm{H}, \mathrm{CH}_{3} \mathrm{CH}_{2}\right.$-porphyrin), $6.20(\mathrm{t}, 1 \mathrm{H}, \mathrm{H}-$ diphenylether), 6.75 (dd, 1H, H-diphenylether), 6.80 (t, 1H, H-diphenylether), 7.06 (dd, 1H, H-diphenylether), 7.17 (d, 1H, H-diphenylether), 7.40 (t, 1H, H-diphenylether), 7.50 ( t, 1H, H-diphenylether), 7.93 (dd, 1H, H-diphenylether), 8.08 (dd, 1H, H-diphenylether), 9.40 (s, 1H, CHO), 9.63 (s, 1H, $\mathrm{H}_{\text {meso }}$-porphyrin), 9.90 (s, 1H, $\mathrm{H}_{\text {meso }}$-porphyrin). MS (MALDI/TOF): $m / z=710.00[\mathrm{M}]^{+\bullet} ; 710.19$ calcd. for $\mathrm{C}_{43} \mathrm{H}_{40} \mathrm{~N}_{4} \mathrm{O}_{2} \mathrm{Zn}$. UV/Vis $\left(\mathrm{CH}_{2} \mathrm{Cl}_{2}\right): \lambda_{\max }\left(\varepsilon \times 10^{-3} \mathrm{Lmol}^{-}\right.$ $\left.{ }^{1} \mathrm{~cm}^{-1}\right)=406.0(336.9), \quad 536.0(17.8), 572.0(15.8) \mathrm{nm}$. Anal. Calcd for $\mathrm{C}_{43} \mathrm{H}_{40} \mathrm{~N}_{4} \mathrm{O}_{2} \mathrm{Zn}: \mathrm{C}$, 72.72; H, 5.68; N, 7.89. Found: C, 72.47; H, 5.95; N, 7.69.

\section{[5-[4-(5,15-Dimesitylcorrol-10-yl)dibenzofuran]-13,17-diethyl-2,3,7,8,12,18-}

hexamethylporphyrin]zinc $\left(\mathbf{P M e s}_{2} \mathbf{C O}\right) \mathbf{Z n H}_{3}$ (14). (Porphyrin)zinc complex (12) (0.369 g, $0.527 \mathrm{mmol}, 1$ equiv. $)$ and mesityldipyrromethane (0.557 g, $2.11 \mathrm{mmol}, 4$ equiv.) were dissolved in $32 \mathrm{~mL}$ of a solution of trifluoacetic acid ( $0.02 \mathrm{mmol}, 0.08$ equiv.) in $\mathrm{CH}_{2} \mathrm{Cl}_{2}(10$ $\mu \mathrm{L}$ of TFA in $100 \mathrm{~mL}$ of $\mathrm{CH}_{2} \mathrm{Cl}_{2}$ ). The reaction mixture was then stirred for $5 \mathrm{~h}$, diluted 25 times with $\mathrm{CH}_{2} \mathrm{Cl}_{2}(800 \mathrm{~mL})$ and a solution of 2,3-dichloro-5,6-dicyano-1,4-benzoquinone (DDQ, $0.360 \mathrm{~g}, 1.581 \mathrm{mmol}, 3$ equiv.) in toluene $(8 \mathrm{~mL})$ was added with stirring. After a further $15 \mathrm{~min}$, the reaction mixture was filtered using a P4 filter, silica gel and $\mathrm{CH}_{2} \mathrm{Cl}_{2}$ as an eluent. The first fraction was collected and concentrated. The violet solid was chromatographed on silica gel eluted with $\mathrm{CH}_{2} \mathrm{Cl}_{2} /$ heptane (60/40). The title compound (14) was obtained in $35 \%$ yield $(0.212 \mathrm{~g}, 0.180 \mathrm{mmol}) .{ }^{1} \mathrm{H}$ NMR $\left(\mathrm{C}_{6} \mathrm{D}_{6}, 300 \mathrm{~K}\right): \delta=0.93(\mathrm{~s}, 6 \mathrm{H}$, $\mathrm{CH}_{3}$-mesityl), 1.48 (s, 6H, $\mathrm{CH}_{3}$-mesityl), 1.83 (t, 6H, $\mathrm{CH}_{3} \mathrm{CH}_{2}$-porphyrin), 2.20 (s, 6H, $\mathrm{CH}_{3}-$ mesityl), 2.25 (s, 6H, $\mathrm{CH}_{3}$-porphyrin), 3.10 (s, 6H, $\mathrm{CH}_{3}-$ porphyrin), 3.21 (s, 6H, $\mathrm{CH}_{3}-$ porphyrin), 3.75 (m, 4H, $\mathrm{CH}_{3} \mathrm{CH}_{2}$-porphyrin), 6.81 (s, 2H, $\mathrm{H}_{\text {meta }}$-mesityl), 7.00 (dd, 1H, Hdibenzofuran), 7.40 (t, 1H, H-dibenzofuran), 7.50 (t, 1H, H-dibenzofuran), 7.23 (s, 2H, $\mathrm{H}_{\text {meta }}-$ mesityl), 7.50 (t, 1H, H-dibenzofuran), 7.53 (dl, 2H, $\mathrm{H}_{\beta}$-corrole), 7.60 (d, 2H, $\mathrm{H}_{\beta}-$ 
corrole), 7.63 (t, 1H, H-dibenzofuran), $7.82\left(\mathrm{t}, 1 \mathrm{H}, \mathrm{H}\right.$-dibenzofuran), $8.00\left(\mathrm{~d}, 2 \mathrm{H}, \mathrm{H}_{\beta}-\right.$ corrole), 8.18 (d, 2H, H $\mathrm{H}_{\beta}$-corrole), 9.07 (s, 2H, $\mathrm{H}_{\text {meso }}$-porphyrin), 9.66 (s, 1H, $\mathrm{H}_{\text {meso }}-$ porphyrin). MS (MALDI/TOF): $m / z=1209.25[\mathrm{M}-3 \mathrm{H}]^{+\bullet} ; 1212.84$ calcd. for $\mathrm{C}_{79} \mathrm{H}_{70} \mathrm{~N}_{8} \mathrm{OZn}$. UV/Vis $\left(\mathrm{CH}_{2} \mathrm{Cl}_{2}\right): \lambda_{\max }\left(\varepsilon \times 10^{-3} \mathrm{Lmol}^{-1} \mathrm{~cm}^{-1}\right)=400.9$ (504.1), $536.0(29.4), 570.0(40.5) \mathrm{nm}$. Anal. Calcd for $\mathrm{C}_{79} \mathrm{H}_{70} \mathrm{~N}_{8} \mathrm{OZn}$ : C, 78.23; H, 5.82; N, 9.24. Found: C, 78.01; H, 5.55; N, 9.06.

\section{[5-[4-(5,15-Dimesitylcorrol-10-yl)diphenylether]-13,17-diethyl-2,3,7,8,12,18-}

hexamethylporphyrin]zinc $\left(\mathrm{PMes}_{2} \mathbf{C O x}\right) \mathrm{ZnH}_{3}$ (13). This compound was synthesized as described above for (14), starting from (porphyrin)zinc complex (11) (0.527 g, 0.745 mmol, 1 equiv.) and mesityldipyrromethane $(0.787 \mathrm{~g}, 2.98 \mathrm{mmol}, 4$ equiv.) in $34 \%$ yield $(0.296 \mathrm{~g}$, $0.250 \mathrm{mmol}) .{ }^{1} \mathrm{H}$ NMR $\left(\mathrm{C}_{6} \mathrm{D}_{6}, 300 \mathrm{~K}\right): \delta=0.91$ (s, 6H, $\mathrm{CH}_{3}-$ mesityl), 1.42 (s, 6H, $\mathrm{CH}_{3^{-}}$ mesityl), 1.65 (t, 6H, $\mathrm{CH}_{3} \mathrm{CH}_{2}-$ porphyrin), 2.34 (s, 6H, $\mathrm{CH}_{3}-$ mesityl), 2.57 (s, 6H, $\mathrm{CH}_{3}-$ porphyrin), 2.73 (s, 6H, $\mathrm{CH}_{3}$-porphyrin), 3.20 (s, 6H, $\mathrm{CH}_{3}-$ porphyrin), 3.30 (m, 4H, $\mathrm{CH}_{3} \mathrm{CH}_{2}$-porphyrin), 6.17 (t, 1H, H-diphenylether), 6.70 (dd, 1H, H-diphenylether), 6.79 (t, 1H, H-diphenylether), 6.81 (s, 2H, $\mathrm{H}_{\text {meta }}$-mesityl), 7.00 (dd, 1H, H-diphenylether), 7.15 (d, 1H, H-diphenylether), 7.20 (s, 2H, $\mathrm{H}_{\text {meta }}$-mesityl), 7.47 (t, 1H, H-diphenylether), 7.49 (d, 2H, $\mathrm{H}_{\beta}$-corrole), 7.80 (d, 2H, ${ }^{3} \mathrm{~J}_{\mathrm{H}-\mathrm{H}}=4.38 \mathrm{~Hz}, \mathrm{H}_{\beta}$-corrole), 7.89 (dd, 1H, H-diphenylether), 8.00 (d, 2H, $\mathrm{H}_{\beta}$-corrole), 8.05 (dd, 1H, H-diphenylether), 8.22 (d, 2H, $\mathrm{H}_{\beta}$-corrole), 9.57 (s, 2H, $\mathrm{H}_{\text {meso }}$-porphyrin), 9.84 (s, 1H, $\mathrm{H}_{\text {meso }}$-porphyrin). MS (MALDI/TOF): $\mathrm{m} / z=1212.75[\mathrm{M}-$ $2 \mathrm{H}]^{+\bullet} ; 1214.86$ calcd. for $\mathrm{C}_{79} \mathrm{H}_{72} \mathrm{~N}_{8} \mathrm{OZn}$. UV/Vis $\left(\mathrm{CH}_{2} \mathrm{Cl}_{2}\right): \lambda_{\max }\left(\varepsilon \times 10^{-3} \mathrm{Lmol}^{-1} \mathrm{~cm}^{-1}\right)=403.0$ (359.0), 536.0 (23.4), 570.0 (28.8) nm. Anal. Calcd for $\mathrm{C}_{79} \mathrm{H}_{72} \mathrm{~N}_{8} \mathrm{OZn}$ : C, 78.10; H, 5.97; N, 9.22. Found: C, 78.47; H, 5.86; N, 9.53.

[2,2'-Bis-(2,8,13,17-tetraethyl-3,7,12,18-tetramethylporphyrin-5-yl)diphenylether]Zn (DPOx)ZnH 2 (17). With a syringe pump, zinc acetate dihydrate $(35.2 \mathrm{mg}, 0.160 \mathrm{mmol})$ dissolved in $10 \mathrm{~mL}$ of absolute methanol was slowly added over 4 hours to a refluxing dichloromethane solution $(250 \mathrm{~mL})$ of $(\mathrm{DPOx}) \mathrm{H}_{4}(\mathbf{1 6})(200 \mathrm{mg}, 0.178 \mathrm{mmol})$. The resulting 
solution was then evaporated, redissolved in dichloromethane and then chromatographed on silica gel using $\mathrm{CH}_{2} \mathrm{Cl}_{2}$ as eluent. The second red band was collected, then evaporated to give (17) in $71 \%$ yield $\left(135 \mathrm{mg}\right.$, based upon $\left.\mathrm{Zn}(\mathrm{OAc})_{2} \cdot 2 \mathrm{H}_{2} \mathrm{O}\right) . \quad{ }^{1} \mathrm{H}$ NMR $\left(\mathrm{CDCl}_{3}\right): \delta .10 .03(\mathrm{~s}$, 2H, meso), 8.04 (d, 2H, ArH), 7.97 (br s, 4H, meso), 7.66 (t, 2H, ArH), 7.09 (t, 2H, ArH), $7.01(\mathrm{~d}, 2 \mathrm{H}, \mathrm{ArH}), 4.31\left(\mathrm{~m}, 4 \mathrm{H},-\mathrm{CH}_{2}-\mathrm{CH}_{3}\right), 4.08\left(\mathrm{~m}, 4 \mathrm{H},-\mathrm{CH}_{2}-\mathrm{CH}_{3}\right), 3.12\left(\mathrm{~s}, 12 \mathrm{H},-\mathrm{CH}_{2^{-}}\right.$ $\left.\mathrm{CH}_{3}\right), 1.86\left(\mathrm{t}, 12 \mathrm{H},-\mathrm{CH}_{2}-\mathrm{CH}_{3}\right), 1.61\left(\mathrm{br}, 12 \mathrm{H},-\mathrm{CH}_{3}\right), 1.58-1.34(\mathrm{~m}, 20 \mathrm{H}),-3.38(\mathrm{~s}, 2 \mathrm{H}, \mathrm{NH})$. MS (MALDI-TOF): $m / z=1186.14[\mathrm{M}]^{+\bullet}, 1186.89$ Calcd. for $\mathrm{C}_{76} \mathrm{H}_{80} \mathrm{~N}_{8} \mathrm{OZn}$. UV-vis $\left(\mathrm{CH}_{2} \mathrm{Cl}_{2}\right), \lambda_{\max }(\mathrm{nm})\left(\varepsilon \times 10^{-4}, \mathrm{~mol}^{-1} 1 \mathrm{~cm}^{-1}\right): 392$ (31.9), 507 (1.0), 537 (1.9), 574 (1.9), 628 (0.1). Anal. Calcd. for $\mathrm{C}_{76} \mathrm{H}_{80} \mathrm{~N}_{8} \mathrm{OZn}$ : C, 76.91; H, 6.79; N, 9.44. Found: C, 76.65; H, 6.57; N, 9.18.

\section{$[2,2$ '-Bis-(2,8,13,17-tetraethyl-3,7,12,18-tetramethylporphyrin-5-}

yl)diphenylether $] Z_{2} \quad$ (DPOx)Zn $\mathbf{Z n}_{2}$ (18). Zinc acetate dihydrate $(200 \mathrm{mg}, 0.91 \mathrm{mmol})$ dissolved in $10 \mathrm{~mL}$ of absolute methanol was added to a chloroform solution $(80 \mathrm{~mL})$ of $(\mathrm{DPOx}) \mathrm{H}_{4}(\mathbf{1 6})(200 \mathrm{mg}, 0.178 \mathrm{mmol})$. The resulting solution was then refluxed during $1 \mathrm{~h}$. After evaporation, the compound was redissolved in dichloromethane, washed 3 times with $250 \mathrm{~mL}$ of $\mathrm{H}_{2} \mathrm{O}$, then dried over $\mathrm{MgSO}_{4}$. The solvent was removed by rotarory evaporation and the crude compound was chromatographed on silica gel using $\mathrm{CH}_{2} \mathrm{Cl}_{2}$ as eluent. The first red band was collected, evaporated to give (18) in quantitative yield $(222 \mathrm{mg}) .{ }^{1} \mathrm{H}$ NMR $\left(\mathrm{CDCl}_{3}\right): \delta .10 .09$ (s, 2H, meso), $8.12(\mathrm{~d}, 2 \mathrm{H}, \mathrm{ArH}), 8.04$ (br s, 4H, meso), 7.75 (t, 2H, ArH), 7.15 (t, 2H, ArH), 7.09 (d, 2H, ArH), $4.41\left(\mathrm{~m}, 4 \mathrm{H},-\mathrm{CH}_{2}-\mathrm{CH}_{3}\right), 4.17$ (m, 4H, $\left.-\mathrm{CH}_{2}-\mathrm{CH}_{3}\right), 3.20$ (s, $\left.12 \mathrm{H},-\mathrm{CH}_{2}-\mathrm{CH}_{3}\right), 1.94\left(\mathrm{t}, 12 \mathrm{H},-\mathrm{CH}_{2}-\mathrm{CH}_{3}\right), 1.71\left(\mathrm{br}, 12 \mathrm{H},-\mathrm{CH}_{3}\right), 1.38-1.24(\mathrm{~m}, 2 \mathrm{H}) . \mathrm{MS}$ (MALDI-TOF): $m / z=1249.53[\mathrm{M}]^{+\bullet}, 1250.27$ Calcd. for $\mathrm{C}_{76} \mathrm{H}_{78} \mathrm{~N}_{8} \mathrm{OZn}$. UV-vis $\left(\mathrm{CH}_{2} \mathrm{Cl}_{2}\right)$, $\lambda_{\max }(\mathrm{nm})\left(\varepsilon \times 10^{-4}, \mathrm{~mol}^{-1} 1 \mathrm{~cm}^{-1}\right): 392$ (40.0), 538 (2.5), 574 (2.4). Anal. Calcd. for $\mathrm{C}_{76} \mathrm{H}_{78} \mathrm{~N}_{8} \mathrm{OZn} 2: \mathrm{C}, 73.01 ; \mathrm{H}, 6.29 ; \mathrm{N}, 8.96$. Found: $\mathrm{C}, 72.95 ; \mathrm{H}, 6.08 ; \mathrm{N}, 9.28$. 

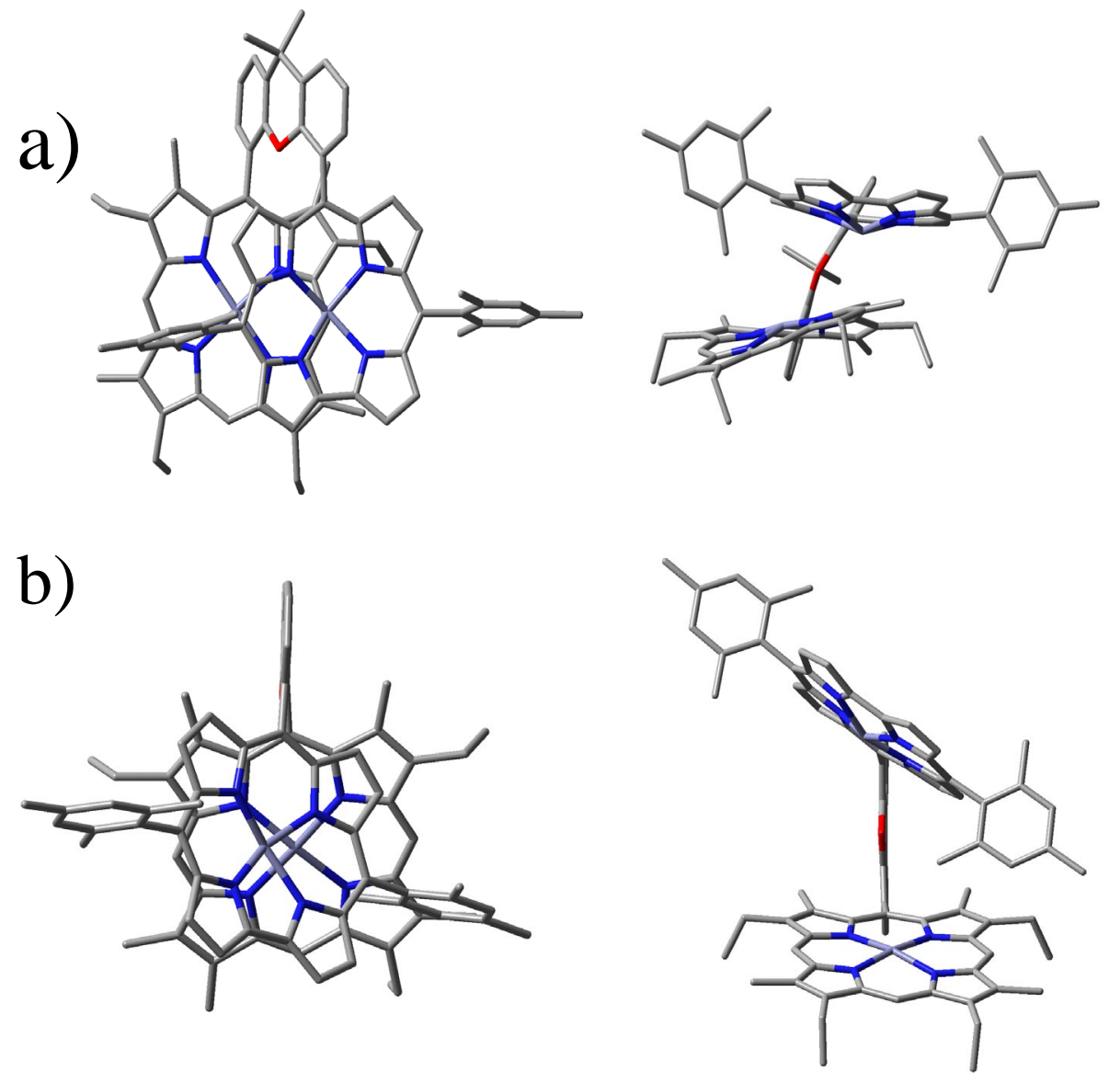

Figure S1. Top and side views of the optimized geometry for $\left(\mathrm{PMes}_{2} \mathrm{CX}\right) \mathrm{ZnH}_{3}$ (15). (top) and for $\left(\mathrm{PMes}_{2} \mathrm{CO}\right) \mathrm{ZnH}_{3}$ (14) (bottom). 
- (Et4Me4Ph1P)H2 -(DPOx)H4

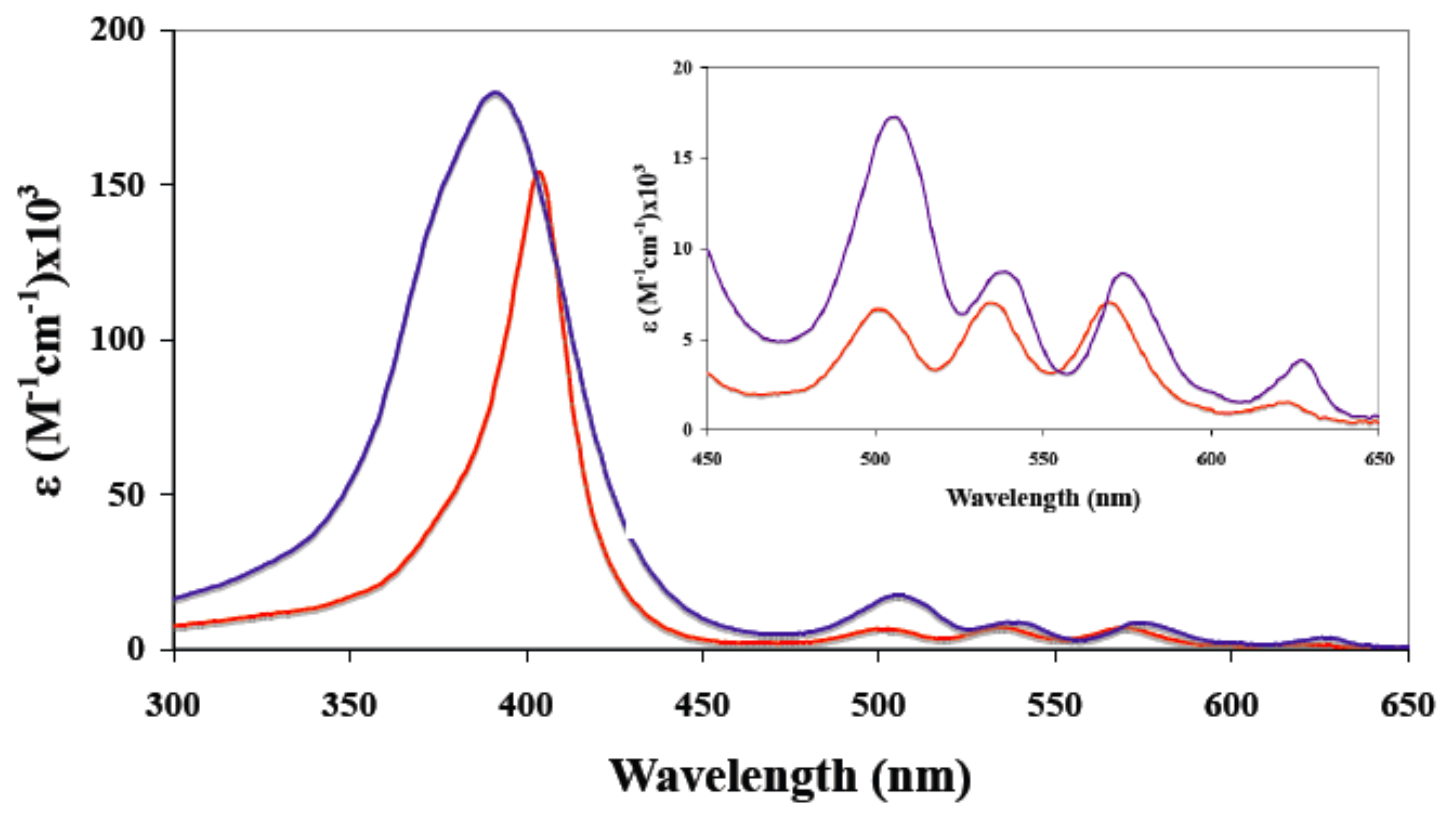

Figure S2. UV-vis spectra of $\left(\mathrm{Et}_{4} \mathrm{Me}_{4} \mathrm{Ph}_{1} \mathrm{P}\right) \mathrm{H}_{2}(7)$ as compared to the free base bisporphyrin (DPOx) $\mathrm{H}_{4}(\mathbf{1 6})$. 
-(DPOx)Zn2 -(Et4Me4Ph1P)Zn X2

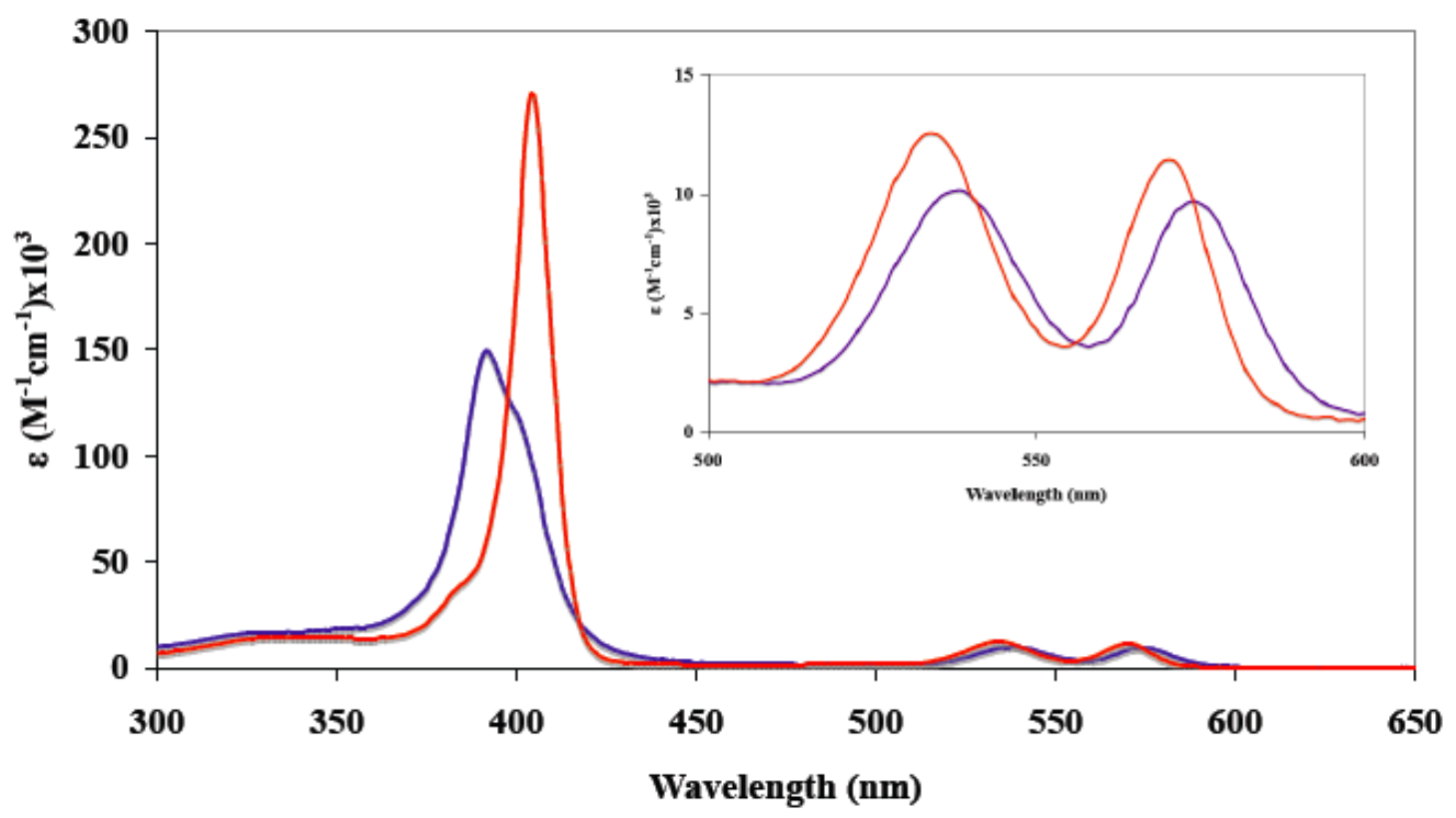

Figure S3. UV-vis spectra of $\left(\mathrm{Et}_{4} \mathrm{Me}_{4} \mathrm{Ph}_{1} \mathrm{P}\right) \mathrm{Zn}(\mathbf{8})$ as compared to the dizinc bisporphyrin (DPOx) $\mathrm{Zn}_{2}(\mathbf{1 8})$. 


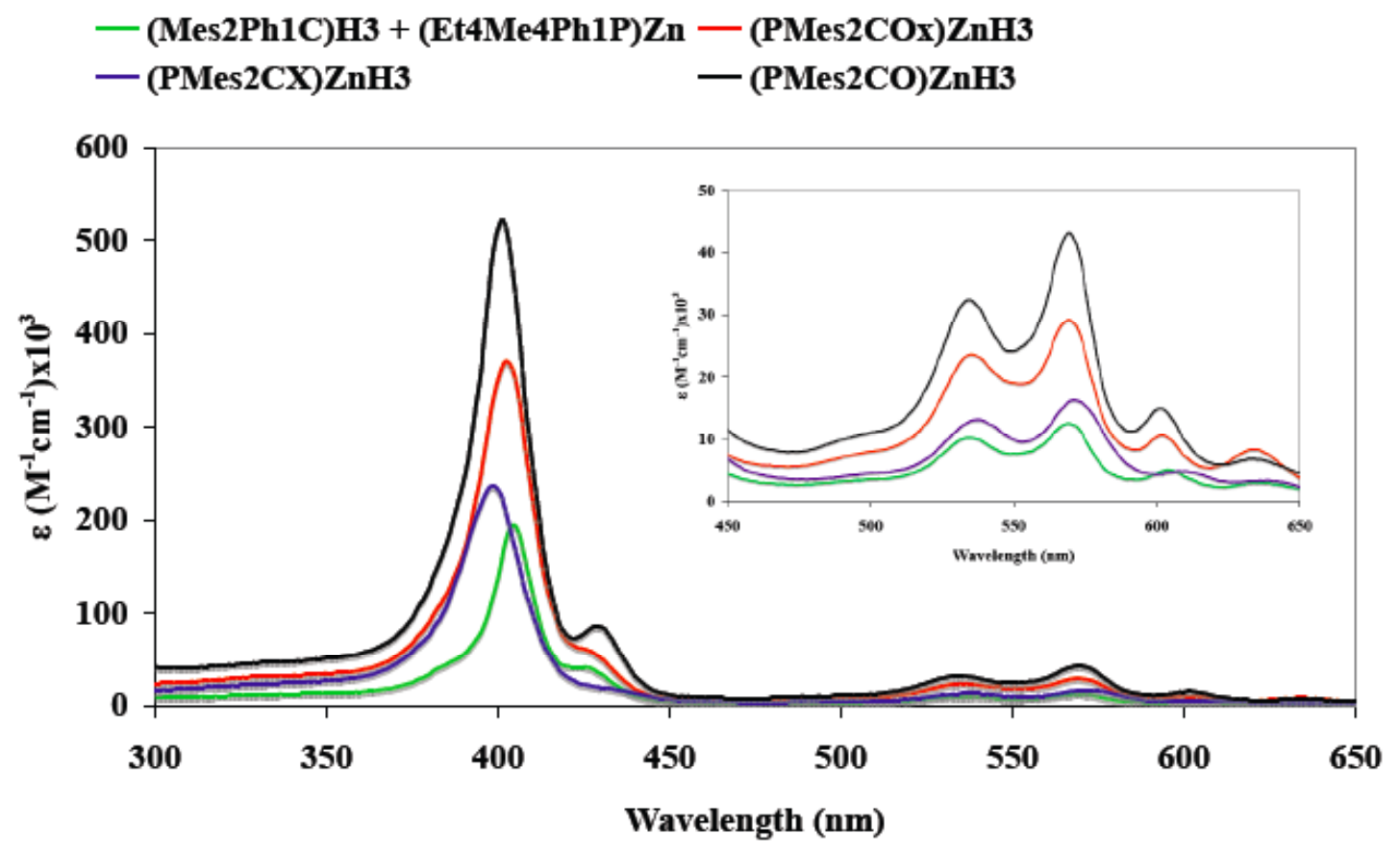

Figure S4. UV-vis spectra of $\left(\mathrm{Mes}_{2} \mathrm{Ph}_{1} \mathrm{Cor}\right) \mathrm{H}_{3}(\mathbf{6})+\left(\mathrm{Et}_{4} \mathrm{Me}_{4} \mathrm{Ph}_{1} \mathrm{P}\right) \mathrm{Zn}(\mathbf{8})$ as compared to the monozinc derivative porphyrin-corroles $\left(\mathrm{PMes}_{2} \mathrm{COx}\right) \mathrm{ZnH}_{3}(\mathbf{1 3}),\left(\mathrm{PMes}_{2} \mathrm{CO}\right) \mathrm{ZnH}_{3}(\mathbf{1 4})$ and $\left(\mathrm{PMes}_{2} \mathrm{CX}\right) \mathrm{ZnH}_{3}(\mathbf{1 5})$. 


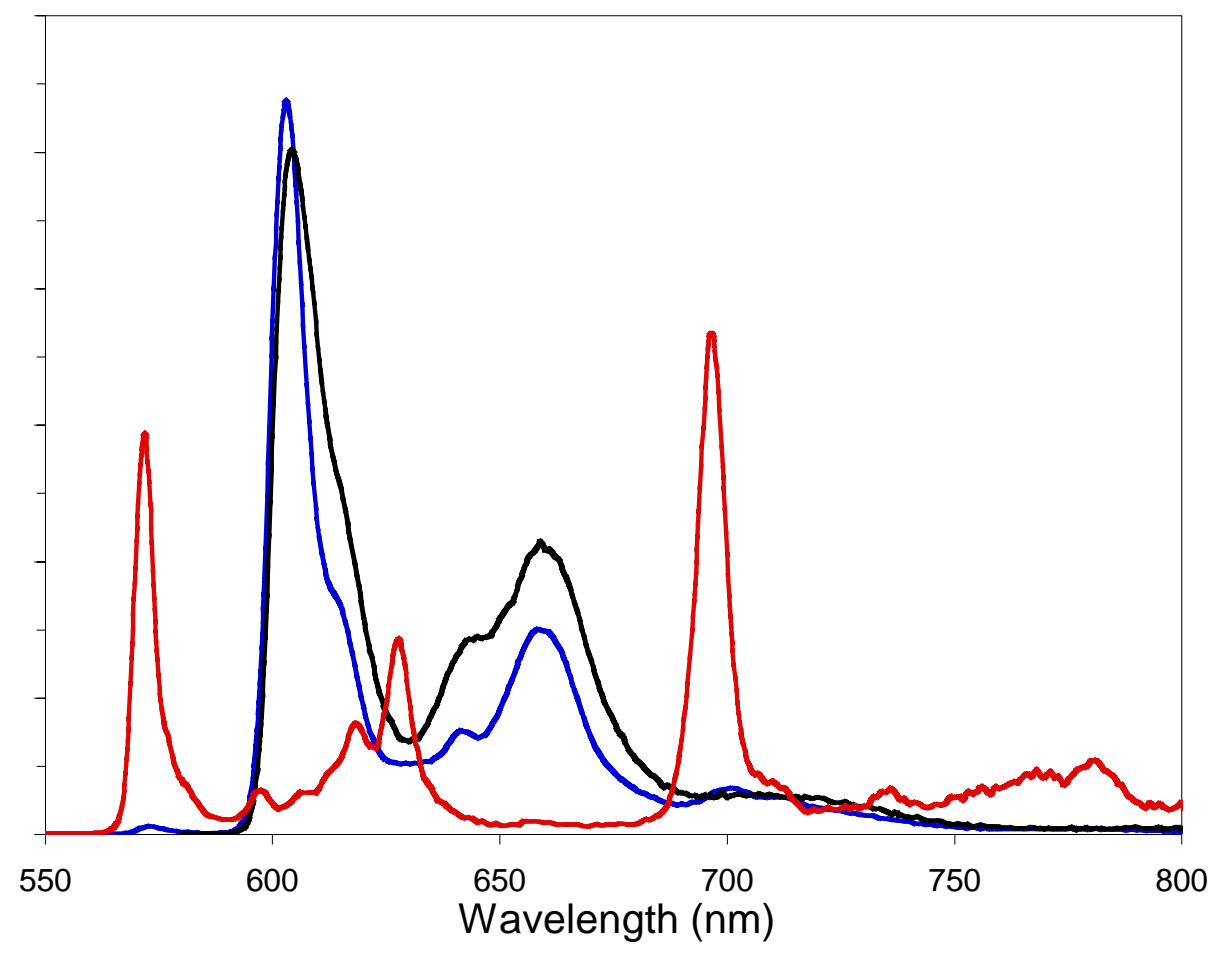

Figure S5. Fluorescence spectra of $\left(\mathrm{PMes}_{2} \mathrm{CO}\right) \mathrm{ZnH}_{3}$ (14) (blue) $\left(\mathrm{Mes}_{2} \mathrm{Ph}_{1} \mathrm{Cor}\right) \mathrm{H}_{3}$ (6) (black) and (OEP)Zn (red) in 2-MeTHF at $77 \mathrm{~K}$. 


\section{Complete Ref. 29}

29. Frisch, M. J.; Trucks, G. W.; Schlegel, H. B.; Scuseria, G. E.; Robb, M. A.; Cheeseman, J. R.; Montgomery, J. A.; Vreven, J., T. ; Kudin, K. N.; Burant, J. C.; Millam, J. M.; Iyengar, S. S.; Tomasi, J.; Barone, V.; Mennucci, B.; Cossi, M.; Scalmani, G.; Rega, N.; Petersson, G. A.; Nakatsuji, H.; Hada, M.; Ehara, M.; Toyota, K.; Fukuda, R.; Hasegawa, J.; Ishida, M.; Nakajima, T.; Honda, Y.; Kitao, O.; Nakai, H.; Klene, M.; Li, X.; Knox, J. E.; Hratchian, H. P.; Cross, J. B.; Adamo, C.; Jaramillo, J.; Gomperts, R.; Stratmann, R. E.; Yazyev, O.; Austin, A. J.; Cammi, R.; Pomelli, C.; Ochterski, J. W.; Ayala, P. Y.; Morokuma, K.; Voth, G. A.; Salvador, P.; Dannenberg, J. J.; Zakrzewski, V. G.; Dapprich, S.; Daniels, A. D.; Strain, M. C.; Farkas, O.; Malick, D. K.; Rabuck, A. D.; Raghavachari, K.; Foresman, J. B.; Ortiz, J. V.; Cui, Q.; Baboul, A. G.; Clifford, S.; Cioslowski, J.; Stefanov, B. B.; Liu, G.; Liashenko, A.; Piskorz, P.; Komaromi, I.; Martin, R. L.; Fox, D. J.; Keith, T.; Al-Laham, M. A.; Peng, C. Y.; Nanayakkara, A.; Challacombe, M.; Gill, P. M. W.; Johnson, B.; Chen, W.; Wong, M. W.; Gonzalez, C.; Pople, J. A. Gaussian 03, Revision C.02,, Gaussian, Inc.: Wallingford CT, 2004. 